\title{
Contextual Mobile Learning System for Saudi Arabian Universities
}

\author{
T. Altameem \\ Dept. of Computer Science, RCC, King Saud University, \\ P.O. Box: 28095 - 11437 Riyadh- Saudi Arabia.
}

\begin{abstract}
With the rapid development of mobile computing technologies, a new style of learning, termed as "mobile learning", has exploded everywhere in the world. This is now considered as an essential learning style in the future. This paper presents the framework of a contextual mobile learning system taking into account the learning environment at Saudi Arabian universities. With the help of this system, the students can learn just in time in their daily lives whenever they need to learn; using mobile computing devices like Tablet PCs and Smart Phones. The main principles and the essential components such as functional modules and context databases are presented. The mobile learning framework has been validated by an interview based qualitative method.
\end{abstract}

Keywords: Mobile Learning, PDAs, Short Message Service (SMS)

\section{INTRODUCTION}

As a result of technological innovations and advancement in computer and instructional technology, we have moved from the chalkboard to television, computer-aided instructions to webbased interactions. The advancements in modern telecommunication capabilities have led to the concept of anytime and anywhere education. However, these are not without limitations. The so-called Internet educational transaction, popularly termed as e-Learning, can only be done 'anywhere' you can find a terminal, and 'anytime' the network is in operation. Contrary to it, the mobile devices have become part of the learner and hence it demolishes the notion of distance and boundaries.

There are certain definitions of mobile education that describe it purely in terms of hardware in a way that it is learning delivered or supported solely or mainly by handheld and mobile technologies such as personal digital assistants (PDAs), smart phones or Tablet PCs. These definitions, however, are confined, techno-centric, and tied to current technological instantiations ${ }^{[1]}$. Let us review the concept of mobile learning, popularly known as m-learning, in a wider context. In today's era, the mobile devices have radically transformed public notions of communication and knowledge. These are now responsible for new forms of entertainment, sports, language, business, policing and learning. If you have used your mobile phone to check the date and time, use the calculator and put in reminders, then you have experienced $\mathrm{m}$-learning as performance support. If you have used the GPS of your mobile phone while driving to ascertain the directions or answered a question posed by your son while you are in office, then you have experienced mobile devices that teach through communication. With enhanced access to information and knowledge anywhere, anytime; the role of education, perhaps especially formal education, is challenged and the relationships between teacher, learner, community, and technology are now more dynamic than ever.

\section{RELATED WORK}

Keegan ${ }^{[2]}$ defined the term m-learning simply as the stipulation of education and training on PDAs, palmtops, smart phones and mobile phones. Trifonova and Ronchetti ${ }^{[3]}$ agreed, noting that $\mathrm{m}$ learning is often defined as e-learning carried out by means of mobile computational devices. They further point out that this refers mainly to PDAs and digital cell phones. Both Quinn ${ }^{[4]}$ and Pinkwart, et al. ${ }^{[5]}$ defined m-learning as "e-learning that uses mobile devices".

Furthermore, the majority of authors, directly or indirectly, apply a definition that views mobile learning as learning connected to a mobile device. To begin with, we shall focus only on the mobile phone as according to Nyiri ${ }^{[6][7]}$ the "... mobile phone is evolving towards the dominant medium. It is becoming the natural interface, through which people conduct their shopping, banking, booking of flights, etc. Moreover, it is turning into the single unique instrument of mediating communication not just between people, but also between people and institutions or more generally between people and the world of inanimate objects".

Georgieva and Smrikarov ${ }^{[8]}$ have presented a general classification of existing mobile learning systems. Hosseini and Mustajärvi ${ }^{[9]}$ have described a framework for mobile learning system based on education component. In a later work, Hosseini $^{[10]}$ defined a framework for mobile learning emphasizing that mobile learning is essentially the evolution of e-learning that completes the missing components of an elearning solution.

Cavus and Momani ${ }^{[11]}$ have described the implementation of a mobile learning system using WiFi network that can act as a huge classroom to make the service available for users in a wide range. Nordin and others ${ }^{[12]}$ proposed a conceptual framework for mobile learning applications for lifelong learning based on four perspectives, namely; generic mobile environment issues, learning contexts, learning experiences and learning objectives. Bates and Poole ${ }^{[13]}$ have proposed a model to effectively utilize the technology for teaching in higher education. The model suggests eight criteria to determine the choice of technology. The analysis of whether the right technology has been chosen is possibly an important feature of a comprehensive assessment of 
mobile learning. It would therefore have to consider the following criteria:

- Only the appropriateness of the technology for students

- $\quad$ Ease of use and reliability

- Costs

- Teaching and learning approaches

- Interactivity

- Organizational issues

- Novelty, as a choice not to use existing technology

- $\quad$ Speed, i.e. How quickly materials can be developed

\section{SAUDI UNIVERSITIES AND THE MOBILE PHONE}

Mobile learning has become a globally accepted phenomenon. Several learning institutions have implemented it in one way or the other. Various assessment studies have ascertained their effectiveness. Georgieva and Smrikarov ${ }^{[14]}$ examined the evaluation of mobile learning system named FLAGMAN developed in the University of Ruse, Bulgaria. The analysis of results of evaluation confirms that the mobile learning system FLAGMAN is technical feasible, didactic effective, cost effective and user friendly. Another analysis done by Nordin and others ${ }^{[15]}$ yields positive results and reveals that adult learners accept m-learning approach. The study also reveals that $\mathrm{m}$ Learning activities are great ways to motivate students and foster interaction among them.

Many of the universities in Saudi Arabia are in practice of utilizing distance learning technologies. Some of the universities have already adopted the Short Message Service (SMS) for teaching and learning. SMS is the text communication service component of phone, web or mobile communication systems, using standardized communications protocols that allow the exchange of short text messages between fixed line or mobile phone devices $^{[16]}$.

For Example, King Saud University has recently initiated a new service that offers users with the ability to send text messages directly from a PC to a mobile phone. The majority of university students have mobile phones and these are increasingly being used to provide other services. The Short Message Service (SMS), or 'texting' as it is sometimes referred as, has become ubiquitous. The primary feature of this facility is its flexibility; the messages can be sent to individual students or groups of students. It does not utilizes the mass marketing approach of an advertising agency, but let the management of faculties, schools or administrative divisions to provide timely, relevant and targeted information to their students.

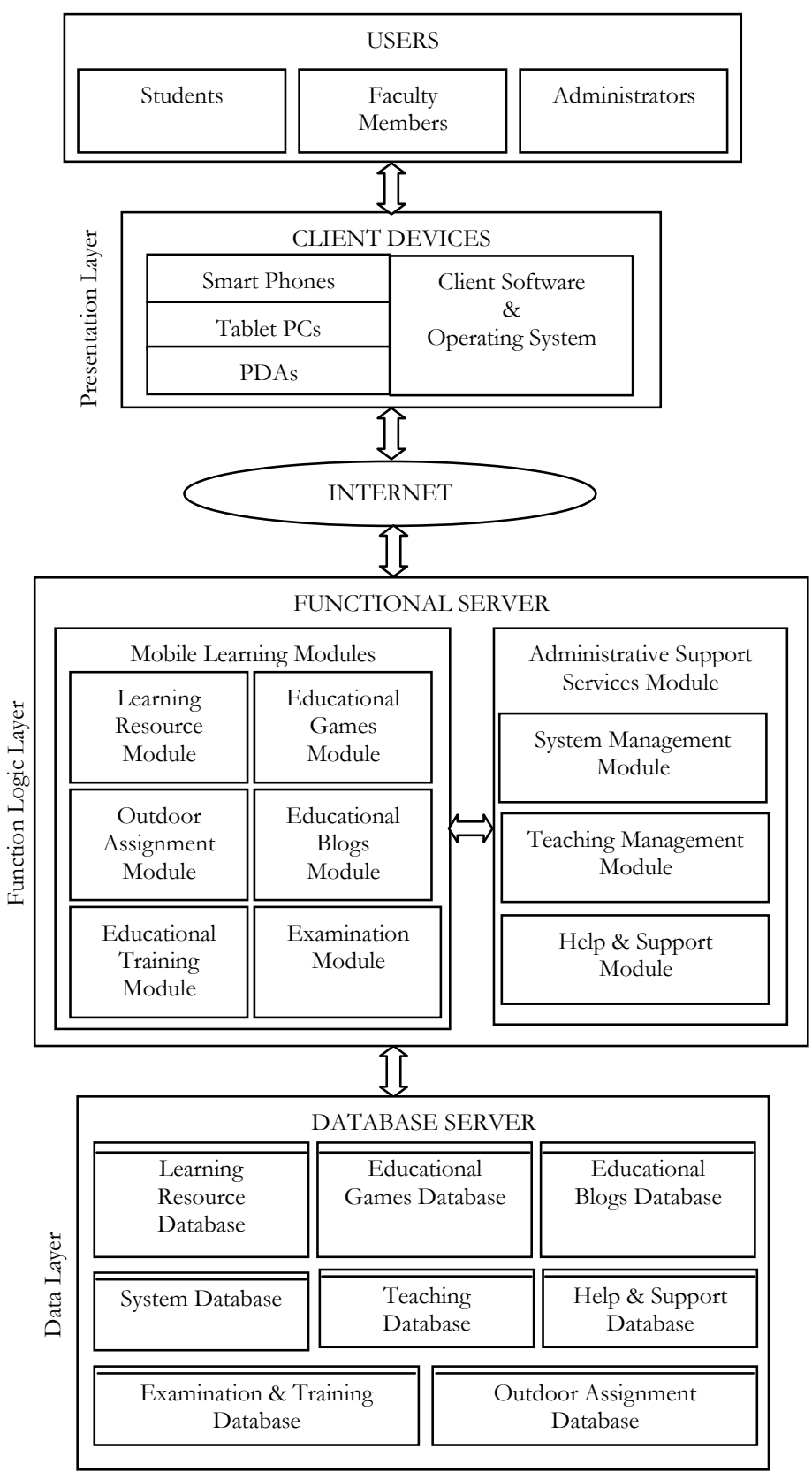

Fig. 1 Framework of Mobile Learning System for KSU

\section{PROPOSED M-LEARNING SYSTEM FOR SAUDI UNIVERSITIES}

Figure 1 describes the framework of Mobile Learning System for Saudi Arabian universities. It is a three tier mobile learning system comprising of Presentation layer, Function logic layer, and the Data layer. The presentation layer would be present in the client devices whereas the function logic layer and data layer are server side layers. 


\subsection{Presentation Layer}

The presentation layer acts as an interactive interface between users and the system. It is implemented on the client devices and its responsibility is to deal with the dialog between users and the system. The client devices are primarily categorized as intelligent mobile phones (Smart phones), PDA, and Tablet PCs. Each of these comprised of their respective hardware and software.

The client application programs of the mobile devices such as operating systems and browsers act as the primary client software for the modules in presentation layer that are using application programs (C/S structure) of smart phones. The browsers of smart phones are used to access data in the mobile learning system server. The browsers run XHTML, WML, and JavaScript to communicate between user requests and server results. Separate user interfaces for students and faculty members are provided by the presentation layer.

\subsection{Data Layer}

Data layer is responsible for providing data sources to the applications. The data layer of the proposed mobile learning system consists of Learning Resource Database, Teaching Database, Outdoor Assignment Database, System Database, Educational Blogs Database, Examination \& Training Database, Help \& Support Database, and Games Database.

The System database stores the basic information of students and teachers. It further contains the processing information of students' learning and faculty member's teaching. The learning resource database consists of mobile learning courseware, electronic lesson plans, e-books, dictionaries and other mobile learning software. The teaching database stores different kinds of educational and administrative information, teaching trends and online courses information such as the name of the class, name of the teacher, the course credits and the course introduction, etc. The outdoor assignment database consists of all kinds of data that is uploaded by the outdoor users. The students can upload their project process and data collected from various sites. The examination and training database has the questions from the students and the answers from the teachers. It also consists of a pool of sample questions that can be used by students for mock-up tests and training. The educational blogs database consists of articles, pictures, criticisms and so on. The games database provides all kinds of educational games to the users.

There is something interesting about learning resource database. It is open to students and is updated according to certain choice mechanism as depicted in Fig. 2:

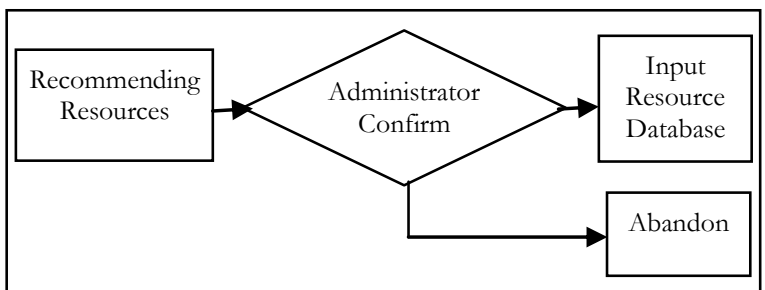

Fig. 2 Choice Mechanism of Leaning

\subsection{Function Logic Layer}

The most important part of the mobile learning system is the function logic layer. All the features of the system are placed in this layer. The function logic layer deals with the application requests of the presentation layer and does logical estimation of the results. The data is then invoked by the system from the related databases; provided the logical estimation is legal. The data layer deals with the invoked data and then returns the results to the presentation layer.

The function logic layer of the mobile learning system consists of two sets of modules: Mobile Learning Modules and Administrative Support Services Modules. The learning modules deals with the students' requests related to learning resources whereas the administrative support services modules deals with the extra-curricular information and administrative support services.

These two modules comprise of nine function modules, namely; System Management Module, Teaching Management Module, Help \& Support Module, Learning Resource Module, Educational Training Module, Outdoor Assignment Module, Examination Module, Educational Blogs Module and Educational Games Module.

\section{FUNCTIONAL LAYER MODULES}

The mobile learning system aims to provide learning services to its users while they are on the move. Hence, the mobile learning system is expected to bear the specific characteristic of the mobile devices - mobility. The mobile learning system should be able to provide particular functions encompassing the principles and theories of mobile learning. The present mobile learning system has been designed with nine viable functions including learning resources, teaching management, help \& support, educational training, outdoor assignment support, Examination, educational Blogs and educational games. Furthermore, the registration and logging functions authorize the students to $\log$ in to the system. The background management function enables the teachers and administrators to manage all the modules of the system. Both of these functions are embedded in the system management module.

In the following sections, we have described the functional modules of our mobile learning system.

\subsection{System Management Module}

The mobile learning system is utilized by three types of users: students, faculty members, and administrators. Initially, the system management module sets and manages the users' registration and authorization. Then the faculty members and administrators manage the whole mobile learning system through the system management module. As existing technologies, the foreground of the system is made up of the operating systems of Smart Devices and the Web application programs of their respective browsers. The faculty members login to the system to deal with the background system and control students information as well as students learning resources. They can upload test and practice resources, supervise the teaching information, and answer students' queries. The administrators enjoy the highest level of authority. They have 
the rights to set and maintain the system besides all of the functions of teachers.

\subsection{Learning Resource Module}

The learning resource module is indeed the most important function of our mobile learning system. The primary objective of the learning resource module is the same as in any e-Learning system. However the mobile learning inculcates the use of mobile devices to carry out learning activities. Hence, we should fully consider that the methods of the mobile learning are individual, spontaneous and portable. We should also consider the hardware and software characteristics of mobile devices so that the course learning functions of the mobile learning system can be designed to have only those information whose contents can be further split into smaller ones. The key objective is to present knowledge in the form of text along with few pictures and animations. While deciding about the nature of mobile learning resources, we should consider some prevailing means such as Web courseware, E-teaching plan, E-book, Electronic dictionary, etc. These would allow students to access mobile learning resources online using web browsers; perform local operations on the accessed resources and download them. Furthermore, the administrators and teachers can upload relevant material and administer the existing mobile learning resources. They also have the privilege to delete and modify access authority.

\subsection{Teaching Management Module}

The teaching management module is vital for the mobile learning system. The design of our mobile learning system offers the following functions.

Functions for students: These include the options to select courses online, view students plan and teaching evaluation, see the results of term end exams, backup the term data, and receive notices for various teaching activities.

Functions for faculty members: These include the insertion of new courses, administering the information about students' course selection, updating information about courses, retrieving the system information, managing the process of teaching evaluation, controlling the process of sending and receiving teaching management information.

Functions for administrators: The administrators have the privileges to alter the functions of teachers' applications, administer the existing courses, manage the process of students' course selection, and broadcast the system news. The administrators also have the rights to manage students' grades, backup score data, and supervise the teaching activities.

\subsection{Educational Training Module}

In the mobile learning system, the learning devices are portable. Hence the learners enjoy the flexibility of learning at various discrete times. These features of mobile learning are most suitable for testing and practice sessions. The mobile educational training module covers these aspects of learning. It offers the following functions:
Functions for students: These include the provision for online testing and practice, providing feedback and score, recording grades, and information about the learning process.

Functions for faculty members: These functions enable the faculty members to setup and upload tests for practice, analyze the grades, evaluate the learning process information, and treat all these as a gist of improving for learners.

\subsection{Outdoor Assignment Module}

The mobile learning system is designed to support the outdoor assignments in a better way due to its portability, communication function, discrete location function and picture function. Hence, the outdoor assignment module appears to be an excellent module of the mobile learning system. This function is particularly suitable for outdoor tasks such as the fieldwork to collect specimen for biological sciences, geography and archaeology. Some of the examples are mentioned below:

- For the research field of biological sciences, the details of the specimen can be recorded, pictured, uploaded and stored.

- For the geographical research field, this function can be used to query users' position information, to acquire accurate GPS coordinates, and to record the local geographical data. Specifically in the exploration of complex terrains, the users are able to carry out the research plan and communicate with each other easily and conveniently.

- For the archaeological research field, the users have the potential to record the information of antiques such as time, place, volume, temperature, pictures and so on by using this function.

\subsection{Educational Games Module}

Learning does not mean consuming more and more knowledge from traditional resources such as books and articles. Researches have confirmed that games can also act as vital resources for learning. To incorporate the concept of learning via entertainment, we have included the module of educational games. The students can utilize this module for interactive learning. Several educational games that develop IQ and language skills can be played by the students. This not only provides entertainment, but also enhances the knowledge skills of students. The faculty members can judge students skills and IQ by the achieved scores. The administrators have the privilege to modify the contents, delete old material and upload new games including the ones proposed by the students.

\subsection{Educational Blogs Module}

Social network sites have become an important aspect of today's networked world. Individual are creating their own blogs and are in contact with each other by means of several e-groups. This module gives an opportunity to our students to remain updated and share knowledge. They can create their own blogs, maintain latest information and can share innovative ideas. The faculty members can judge the creativity of students and support good blogs. The administrators watch that these should not be misused and only relevant educational material be posted on these blogs. 


\subsection{Examination Module}

The examination module helps the students to remain consistent in their academic affairs. They can login to their account and give weekly quizzes. If a student misses a weekly or monthly exam, $\mathrm{s} / \mathrm{he}$ can request the teacher for permission to give the test online at a later date. This simplifies the process of re-exam. The faculty members maintain a large question bank and the exams are conducted by selecting random questions from the database. The teacher only mentions the chapters, types of questions to be asked and the difficulty level of exam. The administrators maintain the steadiness and security of the system.

\subsection{Help and Support Module}

The Help \& Support module is another crucial functional module of the mobile learning system. The key feature of the Help \& Support module is the presence of real-time and intelligent computing. These feature have been made possible due to the portability and communication traits of the mobile devices.

Functions for students: The students are able to ask their respective queries online from the teacher. If a student faces some urgent problems, he may seek teacher's supervision at any normal time using the mobile device. It is not essential to ask the questions only in the designated classrooms at designated time. The Help and Support module can be termed as real- time due to the presence of the following features:

- Automated reply. Most of the possible questions and their respective answers have been uploaded into the FAQ (Frequently Asked Questions) database by the faculty members or administrators in advance. Once a student submits her query, the system searches the databases using the matching keywords retrieval technique. If some related questions are found, the system suggests their answers to the users automatically.

- Browsing questions and answers. The students are able to browse the existing questions and their respective answers using the mobile learning system. They even have the privilege to improve the existing questions and their respective answers.

Functions for faculty members and administrators:

- In case of an emergency situation faced by students, the teachers can answer the questions instantly in the system using the phone or short message service (sms).

- When a certain number of questions are compiled by the students, the system can notify the faculty member to browse.

- The faculty members have the privilege to go through and answer students' queries. They further have the right to store these questions and answers in respective databases.

\section{METHODOLOGY}

The present framework is quite simple and general in nature in a way that it can be applied to any higher education system. However, the framework was specifically designed to suite the demands of higher learning institutions in Saudi Arabia. In order to prove its worthiness for Saudi universities, an interview based qualitative method was utilized. The detailed framework was provided to key figures in the Saudi universities such as deans and other administrative staff in the deanship of Graduate Studies, deanship of e-transaction, deanship of e-learning and distance education, etc. and their views were sought. Most of the participants gave their supporting views. Some of them suggested certain improvements that were included after due discussion.

The following improvements were incorporated as per the suggestions of participants:

a. Separation of function modules into two sets, namely; mobile learning module and administrative support service module.

b. Introduction of educational blog and educational games module.

c. Use of distributed databases.

d. Inclusion of choice mechanism in learning resource module.

\section{FUTURE SCOPE}

The current researches and applications of mobile learning system are still in the evolving phase. Due to this fact and the limits of time and personal capacity, there are several shortcomings in the mobile learning system proposed in this paper. All these limitations can act as the directions for future works in this area.

Although the mobile learning is a powerful application system, the theory researches are still not up to the mark. They have not yet been described precisely. There are certain contradictions that exist in various standard definitions about mobile learning. Hence the present definitions and theories are not précised enough to cope with the developments in the application areas.

The mobile learning system discussed here in this paper requires further research in future. The function logic layer of the mobile learning system does not cover all the necessary aspects. Since the mobile learning is still in its inception phase, the need to add new functions may arise with time. The new applied functions should then be designed to add into the existing framework.

There are certain other things that can be integrated to improve the system. Right now there is no provision to tackle with plagiarism. Since Internet is an open source, everyone has access to online data and can re-publish it with one's own name. Hence some security measures may be taken and certain algorithms may be incorporated in order to deal with it. Another minor issue that can be carried out is to ensure systems' robustness. The provisions to backup the data layer should be taken into account in order to make the system more vigorous.

\section{REFERENCES}

[1] Traxler J., "Defining, Discussing, and Evaluating Mobile Learning: The moving finger writes and having writ... ", The International Review of Research in Open and Distance Learning, Vol 8, No 2 (2007)

[2] Keegan D., "The incorporation of mobile learning into mainstream education and training". Paper presented at mLearn 2005, the 4th World Conference on Mobile Learning held in October 2005 at Cape Town, South Africa. 
[3] Trifonova A. \& Ronchetti M., "A general architecture for M-Learning." Journal of Digital Contents, 2(1) (2003), 3136.

[4] Quinn C. (2000). "mLearning: Mobile, Wireless, In-YourPocket Learning". LiNE Zine. Fall. University of Manchester (undated). The University of Manchester SMS Text Service. Accessed on 13 December 2008 at http://www.itservices.manchester.ac.uk/telecoms/smstextse rvice/

[5] Pinkwart N., Hoppe H.U., Milrad M. \& Perez J., "Educational scenarios for the cooperative use of Personal Digital Assistants". Journal of Computer Assisted Learning, 19(3) (2003), 383-391.

[6] Nyiri K., "Towards a Philosophy of M-Learning". IEEE International Workshop on Wireless and Mobile Technologies in Education (WMTE 2002), August 29-30, 2002, Teleborg Campus

[7] Nyiri K., "The mobile phone in 2005: Where are we now?" Proceedings "Seeing Understanding, Learning in the Mobile Age", Budapest. (2005)

[8] Georgieva E., Smrikarov A. \& Georgiev T., "A General Classification of Mobile Learning Systems", International Conference on Computer Systems and Technologies CompSysTech' 2005

[9] Mostakhdemin-Hosseini A. \& Mustajärvi J., "Framework for mobile learning system based on Education component", Proceedings of the International Conference on Theory and Applications of Mathematics and Informatics - ICTAMI 2003, Alba Iulia

[10] Mostakhdemin-Hosseini A. \& Tuimala J., "Mobile Learning Framework", IADIS International Conference Mobile Learning 2005, ISBN: 972-8939-02-7 (C) 2005 IADIS

[11] Cavus N. \& Al-Momani M.M., "Mobile system for flexible education", Procedia Computer Science, Volume 3, 2011 Pages 1475-1479

[12] Nordin N., Embi M. A. \& Yunus M.M., "Mobile Learning Framework for Lifelong Learning". International conference on Learner Diversity 2010, Procedia - Social and Behavioral Sciences, Volume 7, 2010, Pages 130-138

[13] Bates A.W. \& Poole G., "Effective Teaching with Technology in Higher Education”. San Francisco: JosseyBass (2003).

[14] Georgievaa E.S., Smrikarova A.S. \& Georgieva T.S., "Evaluation of mobile learning system". Procedia Computer Science, Volume 3, 2011, Pages 632-637

[15] Nordin N.M, Hamzah M.I., Yunus M.M. \& Embi M.A., "The Mobile Learning Environment for the In-Service School Administrators". Procedia - Social and Behavioral Sciences, Volume 7, 2010, Pages 671-679

[16] Short Message Service (SMS), http://en.wikipedia.org/wiki/Short_message_service, accessed on December 12, 2010. 